\title{
COMPLETELY REGULAR MONOIDS WITH TWO GENERATORS
}

\author{
MARIO PETRICH \\ (Received 19 November 2009; accepted 26 December 2010) \\ Communicated by M. G. Jackson
}

\begin{abstract}
We classify semigroups in the title according to whether they have a finite or an infinite number of $\mathcal{L}$-classes or $\mathcal{R}$-classes. For each case, we provide a concrete construction using Rees matrix semigroups and their translational hulls. An appropriate relatively free semigroup is used to complete the classification. All this is achieved by first treating the special case in which one of the generators is idempotent. We conclude by a discussion of a possible classification of 2-generator completely regular semigroups.
\end{abstract}

2010 Mathematics subject classification: primary 20M17.

Keywords and phrases: completely regular, monoid, Rees construction, translational hull, ideal extension, generated by, classification.

\section{Introduction and summary}

When studying characterizations of cryptic and of pure semigroups within the class of completely regular semigroups by means of their subsemigroups, we are led to consider completely regular semigroups generated by two elements, $e$ and $b$ say, where $e<b^{0}$ and $e=e^{2}<b$. The classes of cryptogroups and pure completely regular semigroups are characterized by means of classes of concrete semigroups that serve as 'forbidden' subsemigroups. This represents a variant of the method of characterization by means of forbidden divisors. The classes of forbidden subsemigroups are much larger and thus less 'concrete' than the classes of forbidden divisors, but yield more information. This was done in [4] for a class of cryptogroups in terms of a complete list of forbidden subsemigroups. For pure semigroups, this can be found in [3] in terms of homomorphic images of a single forbidden semigroup. As motivation for a treatment of the general case for the former as well as constructing a set of forbidden subsemigroups for the latter, we propose here to study the structure of completely regular semigroups $S$ generated by $\{a, b\}$, where $a<b^{0}$.

(C) 2011 Australian Mathematical Publishing Association Inc. 1446-7887/2011 \$16.00 
We notice first that in such a case $S$ is a monoid with identity $b^{0}$. In this context, it seems natural to attempt to construct all semigroups in the title of this work. This seems a task of intrinsic interest since monogenic completely regular monoids (indeed semigroups) are, trivially, cyclic groups. Two-generator completely regular semigroups have somewhat more complex structure.

\section{Terminology, notation and ideal extensions}

For concepts and symbolism we follow the book [5]. Throughout $S$ denotes an arbitrary completely regular semigroup unless stated otherwise.

Let $S$ be a semigroup. Then $S$ is completely regular if it is a union of its subgroups. If also it contains no proper ideals, it is completely simple. A semilattice is a commutative idempotent semigroup, also considered as a partially ordered set. A completely regular semigroup may be characterized as a semilattice of completely simple semigroups. In detail, $S$ is a disjoint union of completely simple semigroups $S_{\alpha}$, where $\alpha$ runs over a semilattice $Y$ and $S_{\alpha} S_{\beta} \subseteq S_{\alpha \beta}$, for all $\alpha, \beta \in Y$. The semigroups $S_{\alpha}$ are (completely simple) components of $S$.

Now let $S$ be a completely regular semigroup. If Green's $\mathcal{H}$-relation is a congruence on $S$, then $S$ is cryptic or is a cryptogroup. The relation $\leq$ defined on $S$ by

$$
a \leq b \Leftrightarrow a=e b=b f \text { for some idempotents } e \text { and } f \text { in } S
$$

is the natural partial order on $S$.

For emphasis, we list the following notation:

- $E(S)$ denotes the set of idempotents of $S$;

- $S^{0}$ is the semigroup $S$ with a zero adjoined;

- $\quad \mathbb{Z}$ denotes the group of additive integers or its underlying set;

- $\quad \mathbb{Z}_{k}=\mathbb{Z} /(k)$ when $k>1$;

- 1 denotes the identity element of a group;

- If $x$ is an element of a group $G, x^{0}$ denotes the identity of $G$;

- $\langle A\rangle$ stands for the completely regular subsemigroup of $S$ generated by $A$, when $\emptyset \neq A \subseteq S$

- $\langle b\rangle$ denotes the cyclic group generated by $b$;

- $\quad o(b)$ denotes the order of an element $b$;

- $|X|$ stands for the cardinality of a set $X$;

- $\iota_{X}$ denotes the identity map on a set $X$.

If $J$ is an ideal of a semigroup $S$, then $S$ is an (ideal) extension of $J$ by the Rees quotient $Q=S / J$. We will need the following special case of the construction of an ideal extension (see [2, Ch. III, in particular Theorem III.2.5]). Let $J$ be a completely simple semigroup, $B$ be a group disjoint from $J$, and $\sigma$ be a homomorphism of $B$ into the group of units of the translational hull of $J$. On $V=J \cup B$, define a multiplication as follows: when $j \in J$ and $g \in B$, let $g \sigma=(\lambda, \rho)$ and $j * g=j \rho, g * j=\lambda j$, and the other products remain the same. Then $V$ is an extension of $J$ by $B^{0}$, where the identity element of $B$ is the identity of $V$. Conversely, every extension of $J$ by $B^{0}$ with 
this property is isomorphic to a semigroup constructed in this way. For proofs of these statements we refer to the reference cited above. In our case, $J$ will be a Rees matrix semigroup and $B=\langle b\rangle$ a cyclic group generated by an element $b$.

A transformation $\lambda$ (or $\rho$ ) on $S$ written on the left (or right) is a left (or right) translation if $\lambda(x y)=(\lambda x) y$ (or $(x y) \rho=x(y \rho)$ ); if also $x(\lambda y)=(x \rho) y$ for all $x, y \in S$, then $(\lambda, \rho)$ is a bitranslation of $S$. The semigroup $\bar{\Omega}(S)$ of all bitranslations of $S$ under coordinatewise multiplication is the translational hull of $S$. For details concerning this subject, see [2, Section III.1].

Let $I$ and $\Lambda$ be nonempty sets, $G$ be a group, $P$ be a $\Lambda \times I$-matrix over $G$ and $J=\mathcal{M}(I, G, \Lambda ; P)$ be the corresponding Rees matrix semigroup.

For any nonempty set $X$, let $\mathscr{T}(X)$ and $\mathscr{T}^{\prime}(X)$ denote the semigroups of transformations of $X$ written and composed on the left and right respectively. Define the left wreath product as

$$
\mathscr{T}(I) \text { wl } G=\{(\alpha, \varphi) \mid \alpha \in \mathscr{T}(I), \varphi: I \rightarrow G\}
$$

with multiplication

$$
(\alpha, \varphi)\left(\alpha^{\prime}, \varphi^{\prime}\right)=\left(\alpha \alpha^{\prime}, \varphi^{\alpha^{\prime}} \cdot \varphi^{\prime}\right),
$$

where $\varphi^{\alpha^{\prime}} i=\varphi \alpha^{\prime} i$ and $\left(\varphi \cdot \varphi^{\prime}\right) i=(\varphi i)\left(\varphi^{\prime} i\right)$ for all $i \in I$. Dually, define the right wreath product by

$$
G \operatorname{wr} \mathscr{T}^{\prime}(\Lambda)=\left\{(\psi, \beta) \mid \psi: \Lambda \rightarrow G, \beta \in \mathscr{T}^{\prime}(\Lambda)\right\}
$$

with multiplication

$$
(\psi, \beta)\left(\psi^{\prime}, \beta^{\prime}\right)=\left(\psi \cdot{ }^{\beta} \psi^{\prime}, \beta \beta^{\prime}\right),
$$

where $\lambda^{\beta} \psi=\lambda \beta \psi$ and $\lambda\left(\psi \cdot \psi^{\prime}\right)=(\lambda \psi)\left(\lambda \psi^{\prime}\right)$ for all $\lambda \in \Lambda$.

Let

$$
\begin{gathered}
\Omega(J)=\left\{((\alpha, \varphi),(\psi, \beta)) \in(\mathscr{T}(I) \text { wl } G) \times\left(G \text { wr } \mathscr{T}^{\prime}(\Lambda)\right) \mid\right. \\
\left.(\mu \psi) p_{\mu \beta, i}=p_{\mu, \alpha i}(\varphi i) \forall i \in I, \mu \in \Lambda\right\},
\end{gathered}
$$

whose elements we write as $(\alpha, \varphi ; \psi, \beta)$ and give it the direct product multiplication of wreath products. Each $(\alpha, \varphi ; \psi, \beta) \in \Omega(J)$ induces mappings $\lambda$ and $\rho$ on $J$ by

$$
\lambda(i, g, \mu)=(\alpha i,(\varphi i) g, \mu), \quad(i, g, \mu) \rho=(i, g(\mu \psi), \mu \beta),
$$

so that the mapping

$$
\tau:(\alpha, \varphi ; \psi, \beta) \rightarrow(\lambda, \rho) \quad \forall(\alpha, \varphi ; \psi, \beta) \in \Omega(J)
$$

is an isomorphism of $\Omega(J)$ onto $\bar{\Omega}(J)$.

From above we have a homomorphism $\sigma: B \rightarrow \bar{\Omega}(J)$. Set $\theta=\sigma \tau^{-1}$, so that $\theta$ is a homomorphism of $B$ into the group of units of $\Omega(J)$. Since $b$ generates $B$, it suffices to give the image $b \theta$ in $\Omega(J)$ and assume that $b \theta$ generates a subgroup of $\Omega(J)$ whose order divides the order of $b$ if $b$ is of finite order. Therefore

$$
b \theta=(\alpha, \varphi ; \psi, \beta) \in \Omega(J)
$$


and the extension $S$ of $J$ by $B^{0}$ is completely determined by the quadruple $(\alpha, \varphi ; \psi, \beta)$. We shall also need the inverse $\left(\alpha^{\prime}, \varphi^{\prime} ; \psi^{\prime}, \beta^{\prime}\right)$ of $(\alpha, \varphi ; \psi, \beta)$ and an idempotent $a \in J$.

This is our general construction. The parameters are

$$
I, G, \Lambda, P ; \alpha, \varphi, \psi, \beta ; a ; b .
$$

For each case, we shall specify the first nine parameters and then couple this with the case when $b$ is of infinite or of finite order.

We will need the following statement.

LEMMA 2.1. Let $J=\mathcal{M}(I, G, \Lambda ; P)$ and $\theta=(\alpha, \varphi ; \psi, \beta) \in \Omega(J)$.

(i) $\theta$ is the identity of $\Omega(J)$ if and only if

$$
\alpha=\iota_{I}, \quad \varphi i=\lambda \psi=1 \quad \forall i \in I, \lambda \in \Lambda, \quad \beta=\iota_{\Lambda} .
$$

(ii) $\theta$ is in the group of units of $\Omega(J)$ if and only if both $\alpha$ and $\beta$ are permutations.

PROOF. Part (i) follows because

$$
\begin{gathered}
\theta(i, g, \lambda)=(\alpha i,(\varphi i) g, \lambda)=(i, g, \lambda) \Longleftrightarrow \alpha i=i, \varphi i=1, \\
(i, g, \lambda) \theta=(i, g(\lambda \psi), \lambda \beta)=(i, g, \lambda) \Longleftrightarrow \lambda \psi=1, \lambda \beta=\lambda .
\end{gathered}
$$

To see (ii), note that multiplication in the first and the fourth components is coordinatewise. Hence if $\theta$ is in the group of units of $\Omega(J)$, then $\alpha$ and $\beta$ are in the group of units of $\mathscr{T}^{\prime}(I)$ and $\mathscr{T}(\Lambda)$ respectively, so they are permutations.

Conversely, let $\alpha$ and $\beta$ be permutations. Define

$$
\begin{aligned}
\varphi^{\prime} i & =\left(\varphi \alpha^{-1} i\right)^{-1} \quad \forall i \in I, \\
\lambda \psi^{\prime} & =\left(\lambda \beta^{-1} \psi\right)^{-1} \quad \forall \lambda \in \Lambda .
\end{aligned}
$$

Relation (2.1) yields $(\mu \psi) p_{\mu \beta, j}=p_{\mu, \alpha j}(\varphi j)$. Let $\lambda=\mu \beta$ and $i=\alpha j$. Then it follows that $\left(\lambda \beta^{-1} \psi\right) p_{\lambda, \alpha^{-1} i}=p_{\lambda \beta^{-1}, i}\left(\varphi \alpha^{-1} i\right)$, whence

$$
\left(\lambda \beta^{-1} \psi\right)^{-1} p_{\lambda \beta^{-1}, i}=p_{\lambda, \alpha^{-1} i}\left(\varphi \alpha^{-1} i\right)^{-1},
$$

so $\left(\lambda \psi^{\prime}\right) p_{\lambda \beta^{-1}, i}=p_{\lambda, \alpha^{-1} i}\left(\varphi^{\prime} i\right)$ in view of (2.2). Comparing this with (2.1), we see that

$$
\theta^{\prime}=\left(\alpha^{-1}, \varphi^{\prime} ; \psi^{\prime}, \beta^{-1}\right) \in \Omega(J) .
$$

We further obtain

$$
\theta \theta^{\prime}=\left(\iota_{I}, \varphi^{\alpha^{-1}} \cdot \varphi^{\prime} ; \psi \cdot{ }^{\beta} \psi^{\prime}, \iota_{\Lambda}\right)
$$

where

$$
\begin{aligned}
\left(\varphi^{\alpha^{-1}} \cdot \varphi^{\prime}\right) i & =\left(\varphi \alpha^{-1} i\right)\left(\varphi^{\prime} i\right)=1, \\
\lambda\left(\psi \cdot{ }^{\beta} \psi^{\prime}\right) & =(\lambda \psi)\left(\lambda \beta \psi^{\prime}\right)=1,
\end{aligned}
$$


so $\theta \theta^{\prime}=\iota_{\Omega(S)}$ by part (i). Similarly,

$$
\theta^{\prime} \theta=\left(\iota_{I}, \varphi^{\alpha} \cdot \varphi ; \psi^{\prime} \cdot \beta^{-1} \psi, \iota_{\Lambda}\right)
$$

where

$$
\begin{aligned}
\left(\varphi^{\prime \alpha} \cdot \varphi\right) i & =\left(\varphi^{\prime} \alpha i\right)(\varphi i)=1, \\
\lambda\left(\psi^{\prime} \cdot \beta^{-1} \psi\right) & =\left(\lambda \psi^{\prime}\right)\left(\lambda \beta^{-1} \psi\right)=1,
\end{aligned}
$$

and $\theta^{\prime} \theta=\iota_{\Omega(S)}$. Therefore $\theta$ is invertible.

COROLlary 2.2. If $\theta=(\alpha, \varphi ; \psi, \beta)$ is invertible, then $\theta^{-1}=\left(\alpha^{-1}, \varphi^{\prime} ; \psi^{\prime}, \beta^{-1}\right)$, where

$$
\varphi^{\prime} i=\left(\varphi \alpha^{-1} i\right)^{-1}, \quad \lambda \psi^{\prime}=\left(\lambda \beta^{-1} \psi\right)^{-1} \quad \forall i \in I, \lambda \in \Lambda .
$$

PROOF. See the proof of the converse of Lemma 2.1.

\section{The case of $\Gamma$}

In the first part of this section we construct a semigroup, denoted by $\Gamma$, which is an ideal extension of a completely simple semigroup by a cyclic group with a zero adjoined, and establish a number of its properties. Hence we start with the ingredients of the Rees matrix construction. The second part of the section establishes the converse: a semigroup enjoying certain properties will be proved isomorphic to a semigroup of the type constructed in the first part.

Construction 3.1. Let $G$ be a group generated by $\left\{g_{n} \mid n \in \mathbb{Z}\right\}$, where we allow equality of $g_{m}$ and $g_{n}$ even if $m \neq n$. Let $P=\left(p_{n m}\right)$ be the $\mathbb{Z} \times \mathbb{Z}$-matrix:

$$
p_{n m}= \begin{cases}g_{0}^{-1} \cdots g_{n-1}^{-1} g_{m+n-1} \cdots g_{m} & \text { if } n>0, \\ 1 & \text { if } n=0, \\ g_{-1} \cdots g_{n} g_{m+n}^{-1} \cdots g_{m-1}^{-1} & \text { if } n<0,\end{cases}
$$

and let

$$
\Gamma^{*}=\mathcal{M}(\mathbb{Z}, G, \mathbb{Z} ; P) .
$$

Note that $P$ is normalized at 0 .

Next we let $\theta=(\alpha, \varphi ; \psi, \beta)$, where

$$
\alpha n=n+1, \quad \varphi n=g_{n}, \quad n \psi=p_{n 1} g_{0}, \quad n \beta=n+1 \quad \forall n \in \mathbb{Z} .
$$

Explicitly,

$$
n \psi= \begin{cases}g_{0}^{-1} \cdots g_{n-1}^{-1} g_{n} \cdots g_{0} & \text { if } n<0, \\ g_{0} & \text { if } n=0, \\ g_{-1} \cdots g_{n} g_{n+1}^{-1} \cdots g_{-1}^{-1} & \text { if } n<0 .\end{cases}
$$

This is our basic construction. Here is our first result. 
LEMMA 3.2. The quadruple $\theta$ is in the group of units of $\Omega\left(\Gamma^{*}\right)$.

PRoOF. Let $m, n \in \mathbb{Z}$ and

$$
x=p_{n, m+1}(\varphi m), \quad y=(n \psi) p_{n+1, m} .
$$

When $n>0$,

$$
\begin{aligned}
& x=\left(g_{0}^{-1} \cdots g_{n-1}^{-1} g_{m+n} \cdots g_{m+1}\right) g_{m}, \\
& y=\left(g_{0}^{-1} \cdots g_{n-1}^{-1} g_{n} \cdots g_{0}\right)\left(g_{0}^{-1} \cdots g_{n}^{-1} g_{m+n} \cdots g_{m}\right) .
\end{aligned}
$$

When $n=0$,

$$
x=1 \cdot g_{m}, \quad y=g_{0}\left(g_{0}^{-1} g_{m}\right) .
$$

When $n=-1$,

$$
x=\left(g_{-1} g_{m}^{-1}\right) g_{m}, \quad y=g_{-1} \cdot 1 .
$$

When $n<-1$,

$$
\begin{aligned}
& x=\left(g_{-1} \cdots g_{n} g_{m+1+n}^{-1} \cdots g_{m}^{-1}\right) g_{m}, \\
& y=\left(g_{-1} \cdots g_{n} g_{1+n}^{-1} \cdots g_{-1}^{-1}\right)\left(g_{-1} \cdots g_{n+1} g_{m+n+1}^{-1} \cdots g_{m-1}^{-1}\right) .
\end{aligned}
$$

Therefore $x=y$, which proves that $\theta \in \Omega\left(\Gamma^{*}\right)$. Since both $\alpha$ and $\beta$ are permutations, $\theta$ must be invertible by Lemma 2.1(ii), so it is in the group of units of $\Omega\left(\Gamma^{*}\right)$.

Next we provide an explicit expression for the action of $\theta^{k}$ on the left. The same type of argument can be used to compute the right action of $\theta^{k}$.

When $k \in \mathbb{Z}$, we define $\varphi_{k}$ and $\psi_{k}$ implicitly by the formula

$$
\theta^{k}=\left(\alpha^{k}, \varphi_{k} ; \psi_{k}, \beta^{k}\right) \text {. }
$$

LEMMA 3.3. If $k, m, n \in \mathbb{Z}$, then

$$
\begin{aligned}
& \varphi_{k} m= \begin{cases}g_{m+k-1} \cdots g_{m} & \text { if } k>0, \\
1 & \text { if } k=0, \\
g_{m+k}^{-1} \cdots g_{m-1}^{-1} & \text { if } k<0,\end{cases} \\
& n \psi_{k}= \begin{cases}p_{n 1} g_{0} \cdots p_{n+k-1,1} g_{0} & \text { if } k>0, \\
1 & \text { if } k=0, \\
g_{0}^{-1} p_{n+1,1}^{-1} \cdots g_{0}^{-1} p_{n+k, 1}^{-1} & \text { if } k<0 .\end{cases}
\end{aligned}
$$

PROOF. The proof for $\varphi_{k}$ where $k>0$ is by induction. If $k=1$, then $\varphi_{1}=\varphi$, which satisfies the formula. Assume that the formula is true for $k$. Then

$$
\begin{aligned}
\theta^{k+1}(m, g, n) & =\theta\left(\theta^{k}(m, g, n)\right) \\
& =\theta\left(m+k, g_{m+k-1} \cdots g_{m}, n\right) \\
& =\left(m+k+1, g_{m+k} \cdots g_{m}, n\right)
\end{aligned}
$$

and thus $\varphi_{k+1} m=g_{m+k} \cdots g_{m}$. This takes care of the case where $k>0$. Next $\varphi_{0} m=1$ since $\theta^{0}$ is the identity of $\Omega\left(\Gamma^{*}\right)$. 
The argument when $k<0$ is again inductive. By Corollary 2.2,

$$
\begin{aligned}
\theta^{-1}(m, g, n) & =\left(\alpha^{-1} m,\left(\varphi \alpha^{-1} m\right)^{-1} g, n\right) \\
& =\left(m-1,(\varphi(m-1))^{-1} g, n\right) \\
& =\left(m-1, g_{m-1}^{-1} g, n\right),
\end{aligned}
$$

which implies that $\varphi_{-1} m=g_{m-1}^{-1}$ and yields the case where $k=-1$. Assume that the formula is true for $k$. Then

$$
\begin{aligned}
\theta^{k-1}(m, g, n) & =\theta^{-1}\left(\theta^{k}(m, g, n)\right) \\
& =\theta^{-1}\left(m-k, g_{m-k}^{-1} \cdots g_{m-1}^{-1} g, n\right) \\
& =\left(m-k-1,\left(\varphi \alpha^{-1}(m-k)\right)^{-1} g_{m-k}^{-1} \cdots g_{m-1}^{-1} g, n\right) \\
& =\left(m-k-1, g_{m-k-1}^{-1} \cdots g_{m-1}^{-1} g, n\right),
\end{aligned}
$$

and thus $\varphi_{k-1}=g_{m-k-1}^{-1} \cdots g_{m-1}^{-1}$, as required.

The argument for $\psi_{k}$ when $k>0$ is again by induction. If $k=1$, then $\psi_{1}=\psi$, which satisfies the formula. Assume that the formula is true for $k$. Then

$$
\begin{aligned}
(m, g, n) \theta^{k+1} & =\left((m, g, n) \theta^{k}\right) \theta \\
& =\left(m, p_{n 1} g_{0} \cdots p_{n+k-1,1} g_{0}, n+k\right) \theta \\
& =\left(m, p_{n 1} g_{0} \cdots p_{n+k-1,1} g_{0} p_{n+k, 1} g_{0}, n+k+1\right),
\end{aligned}
$$

whence $n \psi_{k+1}=p_{n 1} g_{0} \cdots p_{n+k, 1} g_{0}$, verifying the formula. Since $\theta^{0}$ is the identity of $\Omega\left(\Gamma^{*}\right)$, we see that $n \psi_{0}=1$.

The proof when $k<0$ is also by induction. First

$$
\begin{aligned}
(m, g, n) \theta^{-1} & =\left(m, g\left(n \beta^{-1} \psi\right)^{-1}, n \beta^{-1}\right) \\
& =\left(m, g((n-1) \psi)^{-1}, n-1\right) \\
& =\left(m,\left(p_{n-1,1} g_{0}\right)^{-1}, n-1\right)=\left(m, g_{0}^{-1} p_{n-1,1}^{-1}, n-1\right)
\end{aligned}
$$

which yields the case where $k=-1$. Assume that the formula is true for $k$. Again using Corollary 2.2, we see that

$$
\begin{aligned}
(m, g, n) \theta^{k-1} & =\left((m, g, n) \theta^{k}\right) \theta^{-1} \\
& =\left(m, g g_{0}^{-1} p_{n-1,1}^{-1} \cdots g_{0}^{-1} p_{n-k, 1}^{-1}, n-k\right) \theta^{-1} \\
& =\left(m, g g_{0}^{-1} p_{n-1,1}^{-1} \cdots g_{0}^{-1} p_{n-k, 1}^{-1}((n-k-1) \psi)^{-1}, n-k-1\right) \\
& =\left(m, g g_{0}^{-1} p_{n-1,1}^{-1} \cdots g_{0}^{-1} p_{n-k-1,1}^{-1}, n-k-1\right),
\end{aligned}
$$

which implies that $n \psi_{k-1}=g_{0}^{-1} p_{n-1,1}^{-1} \cdots g_{0}^{-1} p_{n-k-1,1}^{-1}$ and verifies the formula. 
We now introduce some new notation. Let $B=\langle\theta\rangle$ and $B^{0}$ be $B$ with a zero adjoined. Further let $\Gamma$ be the ideal extension of $\Gamma^{*}$ by $B^{0}$ determined by the action of $\theta$, and thus of $\theta^{k}$ when $k \in \mathbb{Z}$, on $\Gamma^{*}$. Finally set

$$
a=(0,1,0) \in \Gamma^{*} .
$$

LEMMA 3.4. As a completely regular semigroup, $\Gamma$ is generated by $\{a, \theta\}$.

PRoOF. Denote by $\Sigma$ the completely regular subsemigroup of $\Gamma$ generated by $\{a, \theta\}$. For all $(m, g, n) \in \Gamma^{*}$, since $P$ is normalized at 0 , we obtain

$$
(m, g, n)=(m, 1,0)(0, g, 0)(0,1, n) .
$$

Also

$$
\left(\theta^{m-1} a\right)^{0}=\left(\theta^{m-1}(0,1,0)\right)^{0}=\left(\alpha^{m} 0,, 0\right)^{0}=(m, 1,0),
$$

and analogously $\left(a \theta^{n-1}\right)^{0}=(0,1, n)$ and thus $(m, 1,0),(0,1, n) \in \Sigma$. By (3.1), it remains to show that $(0, g, 0) \in \Sigma$ for all $g \in G$. Since for all $h_{1}, h_{2}, \ldots, h_{n}, h \in G$, again by normalization, we see that

$$
\begin{gathered}
\left(0, h_{1} h_{2} \cdots h_{n}, 0\right)=\left(0, h_{1}, 0\right)\left(0, h_{2}, 0\right) \cdots\left(0, h_{n}, 0\right), \\
\left(0, h^{-1}, 0\right)^{-1}=(0, h, 0)^{-1},
\end{gathered}
$$

it suffices to show that for all $n \in \mathbb{Z}$, either $\left(0, g_{n}, 0\right) \in \Sigma$ or $\left(0, g_{n}^{-1}, 0\right) \in \Sigma$. If $k, l \in$ $\mathbb{Z}$ and $\varepsilon= \pm 1$, then $\left(0, g_{n}^{\varepsilon}, 0\right)=a\left(k, g_{n}^{\varepsilon}, l\right) a$ and thus it is enough to show that $\left(k, g_{n}, l\right) \in \Sigma$ or $\left(k, g_{n}^{-1}, l\right) \in \Sigma$. Indeed,

$$
\begin{gathered}
\theta a=b(0,1,0)=(\alpha 0, \varphi 0,0)=\left(1, g_{0}, 0\right), \\
\left(\theta^{2} a\right)(\theta a)^{-1}=b\left(1, g_{0}, 0\right)\left(1, g_{0}, 0\right)^{-1}=\left(2,(\varphi 1) g_{0}, 0\right)\left(1, g_{0}^{-1}, 0\right)=\left(2, g_{1}, 0\right),
\end{gathered}
$$

and continuing this procedure, we see that $\left(n, g_{n-1}, 0\right) \in \Sigma$ for all $n>0$. Similarly

$$
\begin{aligned}
\theta^{-1}= & \left(\alpha^{-1} 0,\left(\varphi \alpha^{-1} 0\right)^{-1}, 0\right)=\left(-1, g_{-1}^{-1}, 0\right), \\
\left(\theta^{-2} a\right)\left(\theta^{-1} a\right)^{-1} & =b^{-1}\left(-1, g_{-1}^{-1}, 0\right)\left(-1, g_{-1}^{-1}, 0\right) \\
& =\left(\alpha^{-1}(-1),\left(\varphi \alpha^{-1}(-1)\right)^{-1} g_{-1}^{-1}, 0\right)\left(1, g_{-1}, 0\right) \\
& =\left(-2, g_{-2}^{-1} g_{-1}^{-1}, 0\right)\left(1, g_{-1}, 0\right)=\left(-2, g_{-2}^{-1}, 0\right) .
\end{aligned}
$$

Continuing this procedure, we obtain $\left(-n, g_{-n}^{-1}, 0\right) \in \Sigma$ for all $n>0$. Therefore $\Gamma \subseteq \Sigma$ and the equality prevails.

We may summarize the main properties of $\Gamma$ :

- $\quad \Gamma$ is a completely regular semigroup with components $\Gamma^{*}$ and $B$;

- $\quad \theta^{0}$ is the identity element of $\Gamma$;

- $a<\theta^{0}$;

- $\quad\{a, \theta\}$ generates $\Gamma$ as a completely regular semigroup;

- $\quad \Gamma$ has infinitely many $\mathcal{L}$-classes and $\mathcal{R}$-classes. 
We now turn to the converse. Let $S=\left(Y ; S_{\alpha}\right)$ be a completely regular semigroup generated by $\left\{a, b^{0}\right\}$, where $a \in E\left(S_{\alpha}\right), b \in S_{\beta}, a<b$ and $S$ has infinitely many $\mathcal{L}$ classes and $\mathcal{R}$-classes. It follows that $Y=\{\alpha, \beta\}$ where $\alpha<\beta$; let $A=S_{\alpha}$ and $B=S_{\beta}$. We may set

$$
A=\mathcal{M}(I, G, \Lambda ; P), \quad a=(0,1,0),
$$

where we assume that $P$ is normalized at 0 . Then $B$ is a cyclic group generated by $b$. The action of $b$ on $A$ induces a bitranslation, $\theta$ say, of $A$. We have assumed that $a<b^{0}$, which implies that $b^{0}$ is the identity of $S$.

We write the bitranslation $\theta$ as a quadruple $(\alpha, \varphi ; \psi, \beta)$ and must solve the equation

$$
p_{\lambda, \alpha i}(\varphi i)=(\lambda \psi) p_{\lambda \beta, i}
$$

for $p, \varphi$ and $\beta$ in terms of the generators of $G$. Clearly $I$ must be the orbit of $\alpha$ at 0 , and $\Lambda$ the orbit of $\beta$ at 0 .

We have assumed that $S$ has infinitely many $\mathcal{L}$-classes and $\mathcal{R}$-classes. This means that both $I$ and $\Lambda$ are infinite and thus both functions $\alpha$ and $\beta$ are of infinite order. It follows that we may set $I=\Lambda=\mathbb{Z}$, where

$$
\alpha, \beta: n \rightarrow n+1 \quad \forall n \in \mathbb{Z} .
$$

Since $\alpha$ and $\beta$ are of infinite order, so is $\theta$. It follows that the homomorphism induced by the function $b \rightarrow \theta$ is injective and is thus an isomorphism of $B$ onto $\langle\theta\rangle$. Hence we need not consider $\langle b\rangle$ but $\langle\theta\rangle$ will do instead.

First equation (3.2) in our new notation takes on the form

$$
p_{n, m+1}(\varphi m)=(n \psi) p_{n+1, m} \quad \forall m, n \in \mathbb{Z} .
$$

We now define

$$
g_{m}=\varphi m \quad \forall m \in \mathbb{Z},
$$

and let $G=\left\langle g_{m} \mid m \in \mathbb{Z}\right\rangle$ be the group generated by the $g_{m}$. Hence

$$
A=\mathcal{M}(\mathbb{Z}, G, \mathbb{Z} ; P),
$$

where $P$ still has to be determined. In (3.3), we have $\alpha$ and $\beta$, so it remains to calculate $\psi$.

Setting $m=n=0$ in (3.4) and taking into account (3.5), we see that $g_{0}=\varphi 0=0 \psi$. Again in (3.4), letting $m=0$, we obtain

$$
n \psi=p_{n 1} g_{0} \quad \forall n \in \mathbb{Z} .
$$

It thus remains to determine the entries of $P$. Now (3.4) becomes

$$
p_{n, m+1} g_{m}=p_{n 1} g_{0} p_{n+1, m} \quad \forall m, n \in \mathbb{Z} .
$$

We claim that

$$
p_{n m}=g_{0}^{-1} \cdots g_{n-1}^{-1} g_{m+n-1} \cdots g_{m} \quad \forall n>0, m \in \mathbb{Z} .
$$

The proof is by induction on $n$. When $n=0$, relation (3.8) yields $p_{0, m+1} g_{m}=$ $p_{01} g_{0} p_{1 m}$, whence $g_{0}^{-1} g_{m}=p_{1 m}$, which is the case where $n=1$ in (3.9). Assume 
the validity of (3.9). Using (3.4), (3.5) and (3.7), we obtain

$$
\begin{aligned}
p_{n+1, m} & =(n \psi)^{-1} p_{n, m+1}(\varphi m) \\
& =\left(g_{0}^{-1} p_{n 1}^{-1}\right)\left(g_{0}^{-1} \cdots g_{n-1}^{-1} g_{m+n} \cdots g_{m+1}\right) g_{m} \\
& =g_{0}^{-1}\left(g_{0}^{-1} \cdots g_{n-1}^{-1} g_{n} \cdots g_{1}\right)^{-1}\left(g_{0}^{-1} \cdots g_{n-1}^{-1} g_{m+n} \cdots g_{m}\right) \\
& =g_{0}^{-1} g_{1}^{-1} \cdots g_{n}^{-1} g_{n-1} \cdots g_{0} g_{0}^{-1} \cdots g_{n-1}^{-1} g_{m+n} \cdots g_{m} \\
& =g_{0}^{-1} \cdots g_{n}^{-1} g_{m+n} \cdots g_{m},
\end{aligned}
$$

which establishes the claim.

We also claim that

$$
p_{-n, m}=g_{-1} \cdots g_{-n} g_{m-n}^{-1} \cdots g_{m-1}^{-1} \quad \forall n>0, m \in \mathbb{Z} .
$$

The proof is by induction on $n$. For the case where $n=1$, in (3.8) we first let $n=-1$, getting $p_{-1, m+1}=p_{-1,1} g_{0} g_{m}^{-1}$, and then also $m=-1$, obtaining $1=p_{-1,1} g_{0} g_{-1}^{-1}$. These last two equations imply that

$$
p_{-1, m+1}=g_{-1} g_{0}^{-1} g_{0} g_{m}^{-1}=g_{-1} g_{m}^{-1},
$$

which satisfies (3.10). Now assume the validity of (3.10). From (3.8),

$$
p_{-(n+1), m} g_{m-1}=p_{-(n+1), 1} g_{0} p_{-n, m-1} .
$$

When $m=0$, this gives

$$
\begin{aligned}
p_{-(n+1), 1} & =g_{-1} p_{-n,-1}^{-1} g_{0}^{-1} \\
& =g_{-1}\left(g_{-1} \cdots g_{-n} g_{-1-n}^{-1} \cdots g_{-2}^{-1}\right)^{-1} g_{0}^{-1} \\
& =g_{-1} g_{-2} \cdots g_{-1-n} g_{-n}^{-1} \cdots g_{-1}^{-1} g_{0}^{-1},
\end{aligned}
$$

which together with (3.11) yields

$$
\begin{aligned}
p_{-(n+1), m} & =\left(g_{-1} \cdots g_{-1-n} g_{-n}^{-1} \cdots g_{-1}^{-1} g_{0}^{-1}\right) g_{0}\left(g_{-1} \cdots g_{-n} g_{m-1-n}^{-1} \cdots g_{m-2}^{-1}\right) g_{m-1}^{-1} \\
& =g_{-1} \cdots g_{-(n+1)} g_{m-(n+1)}^{-1} \cdots g_{m-1}^{-1},
\end{aligned}
$$

as required.

From (3.9) and (3.10),

$$
p_{n m}= \begin{cases}g_{0}^{-1} \cdots g_{n-1}^{-1} g_{m+n-1} \cdots g_{m} & \text { if } n>0 \\ 1 & \text { if } n=0 \\ g_{-1} \cdots g_{n} g_{m+n}^{-1} \cdots g_{m-1}^{-1} & \text { if } n<0 .\end{cases}
$$

From (3.5) and (3.7), we see that

$$
\varphi_{m}=g_{m}, \quad n \psi= \begin{cases}g_{0}^{-1} \cdots g_{n-1}^{-1} g_{n} \cdots g_{0} & \text { if } n>0, \\ g_{n} & \text { if } n=0,-1, \\ g_{-1} \cdots g_{n} g_{1+n}^{-1} \cdots g_{-1}^{-1} & \text { if } n<-1 .\end{cases}
$$

We have proved that $S$ is of type $\Gamma$. 


\section{The case of $\Gamma_{\ell}$}

We now modify Construction 3.1 as follows. Let $\ell>0$ and let

$$
I_{\ell}=\{0, \ldots, \ell-1\}, \quad G_{\ell}=\left\{g_{0}, \ldots, g_{\ell-1}\right\} .
$$

Extend the sequence $g_{0}, \ldots, g_{\ell-1}$ to the sequence $\left\{g_{n} \mid n \in \mathbb{Z}\right\}$ with the proviso that

$$
g_{m}=g_{n} \Longleftrightarrow m \equiv n \quad \bmod \ell
$$

Now let $\Gamma_{\ell}^{*}=\mathcal{M}\left(I_{\ell}, G_{\ell}, \mathbb{Z} ;\left.P\right|_{\mathbb{Z} \times I_{\ell}}\right)$, let $\alpha_{\ell}=(0 \cdots \ell-1)$ be a cycle, and let $\theta_{\ell}=$ $\left(\alpha_{\ell},\left.\varphi\right|_{I_{\ell}} ; \psi, \beta\right)$. Since $\beta$ is of infinite order, so is $\theta_{\ell}$.

LEMMA 4.1. The quadruple $\theta_{\ell}$ is in the group of units of $\Omega\left(\Gamma_{\ell}^{*}\right)$.

PROOF. We could mimic the proof of Lemma 3.2 with additional complications because of the finiteness of $I_{\ell}$. Instead, we adopt the result of that lemma but must additionally show that $p_{n, m \ell}=1$ for all $m \geq 0$. First

$$
p_{n, m \ell}= \begin{cases}g_{0}^{-1} \cdots g_{n-1}^{-1} g_{m \ell+n-1} \cdots g_{m \ell} & \text { if } n>0 \\ 1 & \text { if } n=1, \\ g_{-1} \cdots g_{n} g_{m \ell+n}^{-1} \cdots g_{m \ell-1}^{-1} & \text { if } n<0 .\end{cases}
$$

Taking into account our convention that $g_{m \ell+s}=g_{s}$ for all choices of $m, s \geq 0$, we see that

$$
p_{n, m \ell}= \begin{cases}g_{0}^{-1} \cdots g_{n-1}^{-1} g_{n-1} \cdots g_{0} & \text { if } n>0 \\ 1 & \text { if } n=0 \\ g_{-1} \cdots g_{n} g_{n}^{-1} \cdots g_{-1}^{-1} & \text { if } n<0\end{cases}
$$

whence $p_{n, m \ell}=1$. Since both $\alpha_{\ell}$ and $\beta$ must be permutations, by Corollary 2.2, we see that $\theta_{\ell}$ is in the group of units of $\Omega\left(\Gamma_{\ell}^{*}\right)$.

As in the preceding section, we obtain a semigroup $\Gamma_{\ell}$, now with $(\ell+1) \mathcal{R}$-classes. In addition to Lemma 4.1, we may prove, in essentially the same way, the analogues of Lemmas 3.3 and 3.4.

Conversely, we now assume that $S$ satisfies the conditions in the converse part in the preceding section except that it now has finitely many $\mathcal{R}$-classes. This is the case when the orbit of $\alpha$ is finite and the orbit of $\beta$ remains infinite. Hence

$$
I=\{0, \ldots, \ell-1\}, \quad \ell>0, \quad \Lambda=\mathbb{Z} .
$$

It follows that $p_{n, m \ell}=1$ for all $n, m \in \mathbb{Z}$. We may thus use the formula for $p_{n m}$ in the preceding section, so that when $n>0$,

$$
g_{0}^{-1} \cdots g_{n-1}^{-1} g_{m \ell+n-1} \cdots g_{m \ell}=1,
$$


that is, $g_{n-1} \cdots g_{0}=g_{m \ell+n-1} \cdots g_{m \ell}$. For

$$
\begin{aligned}
& n=1: g_{0}=g_{m \ell}, \\
& n=2: g_{1} g_{0}=g_{m \ell+1} g_{m \ell} \Rightarrow g_{1}=g_{m \ell+1}, \\
& n=3: g_{2} g_{1} g_{0}=g_{m \ell+2} g_{m \ell+1} g_{m \ell} \Rightarrow g_{2}=g_{m \ell+2}
\end{aligned}
$$

and continuing, we see that $g_{n}=g_{m \ell+n}$. Since $\varphi m=g_{m}$ if $m=0, \ldots, \ell-1$, we deduce that $G=\left\langle g_{0}, \ldots, g_{\ell-1}\right\rangle$.

Therefore $S$ is of type $\Gamma_{\ell}$.

\section{The case of $\Gamma_{\ell}$}

In addition to the modification of Construction 3.1 in the preceding section, we now let $\Lambda_{r}=\{0, \ldots, r-1\}$ and $\beta_{r}=(0 \cdots r-1)$ be a cycle, where $r>0$. We extend the sequence $g_{0}, \ldots, g_{r-1}$ to $\left\{g_{n} \mid n \in \mathbb{Z}\right\}$ as in the preceding section; see (4.2). We also impose the following condition:

$$
g_{r-1} \cdots g_{0}=g_{m+r-1} \cdots g_{m} \quad \forall m=1, \ldots, \ell-1 .
$$

Recall that in the preceding section we set

$$
I_{\ell}=\{0, \ldots, \ell-1\}, \quad G_{\ell}=\left\{g_{0}, \ldots, g_{\ell-1}\right\}
$$

see (4.1). Let $\gamma$ be the congruence on $G_{\ell}$ induced by (5.1) and let $G_{\ell r}=G_{\ell} / \gamma$. That is, letting $N$ be the normal subgroup of $G_{\ell}$ generated by

$$
\left\{g_{r-1} \cdots g_{0}\left(g_{m+r-1} \cdots g_{m}\right)^{-1} \mid m=1, \ldots, \ell-1\right\},
$$

we have $G_{\ell r}=G_{\ell} / N$.

Define

$$
\begin{gathered}
\Gamma_{\ell r}^{*}=\mathcal{M}\left(I_{\ell}, G_{\ell r}, \Lambda_{r} ;\left.P\right|_{\Lambda_{r} \times I_{\ell}}\right), \\
\theta_{\ell r}=\left(\alpha_{\ell},\left.\varphi\right|_{I_{\ell}} ;\left.\psi\right|_{\Lambda_{r}}, \beta_{r}\right) .
\end{gathered}
$$

We now go through the procedure adopted in the preceding two sections.

LEMMA 5.1. The quadruple $\theta_{\ell r}$ is in the group of units of $\Omega\left(\Gamma_{\ell r}^{*}\right)$.

PROOF. In view of Lemma 4.1, which handled the case of $\theta_{\ell}$, it now remains to prove that

$$
p_{n r, m}=1 \quad \forall n, m>0 .
$$

We fix $m$ and prove the formula by induction on $n$. Note that (5.2) is equivalent to

$$
g_{n r-1} \cdots g_{0}=g_{m+n r-1} \cdots g_{m} \quad \forall m>0 .
$$


By (5.1), formula (5.3) holds if $n=1$. Assume that it holds for $n$; then

$$
\begin{aligned}
g_{(n+1) r-1} \cdots g_{0} & =g_{r+n r-1} \cdots g_{0} \\
& =\left(g_{r+n r-1} \cdots g_{r}\right)\left(g_{r-1} \cdots g_{0}\right) \\
& =\left(g_{m+r+n r-1} \cdots g_{m+r}\right)\left(g_{m+r-1} \cdots g_{m}\right) \\
& =g_{m+(n+1) r-1} \cdots g_{m},
\end{aligned}
$$

completing the proof of (5.3). Hence $\theta_{\ell r} \in \Omega\left(\Gamma_{\ell r}^{*}\right)$ and since both functions $\alpha$ and $\beta$ are permutations, by Corollary 2.2, $\theta_{\ell r}$ is in the group of units of $\Omega\left(\Gamma_{\ell r}^{*}\right)$.

This is an analogue of Lemma 3.2; analogues of Lemmas 3.3 and 3.4 follow similarly.

Conversely, we assume that $S$ satisfies the conditions in the converse part of Section 3 (or 4 ), except that now it has only a finite number of both $\mathcal{L}$-classes and $\mathcal{R}$-classes, that is, both $\alpha$ and $\beta$ are of finite order. Hence

$$
I=\{0, \ldots, \ell-1\}, \quad \Lambda=\{0, \ldots, r-1\},
$$

where $\ell, r>0$. In view of the preceding case, $p_{n, m \ell}=p_{n r, m}=1$ for all $n, m \in \mathbb{Z}$. In the formula for $p_{n m}$, when $m>0$,

$$
g_{0}^{-1} \cdots g_{n r-1}^{-1} g_{m+n r-1} \cdots g_{m}=1
$$

that is, $g_{n r-1} \cdots g_{0}=g_{m+n r-1} \cdots g_{m}$. In particular, this is valid if $n=1$ and $m=$ $1, \ldots, r-1$, giving

$$
g_{r-1} \cdots g_{0}=g_{m+r-1} \cdots g_{m} \quad \forall m=1, \ldots, k-1 .
$$

We have proved that $S$ is of type $\Gamma_{\ell} r$.

In all three cases considered, the conditions obtained ensure the existence of an ideal extension of the completely simple semigroup $\Gamma^{*}, \Gamma_{\ell}^{*}$ or $\Gamma_{\ell r}^{*}$ by the cyclic group $\langle\theta\rangle,\left\langle\theta_{\ell}\right\rangle$ or $\left\langle\theta_{\ell r}\right\rangle$ with a zero adjoined, respectively. For the first two cases, $\theta$ and $\theta_{\ell}$ are of infinite order, so that the homomorphism (the extension function) $B \rightarrow\langle\theta\rangle$ or $\left\langle\theta_{\ell}\right\rangle$ is an isomorphism. This need not be the case in the third instance.

We now expand the construction of $\Gamma_{\ell r}$ to include an ideal extension of $\Gamma_{\ell r}^{*}$ by a cyclic group with zero. We start by letting $B$ be a cyclic group of order $s$ (finite or infinite) generated by an element $b$. By [4, Corollary 5.3], in order that a homomorphism of $B$ onto $\langle\theta\rangle$ exists, it is necessary and sufficient that if $o(b)=s>0$, then

$$
s=p \ell=q r, \quad\left(g_{\ell-1} \cdots g_{0}\right)^{p}=\left(g_{r-1} \cdots g_{0}\right)^{q}=1,
$$

for some positive integers $p$ and $q$. We assume the validity of this condition.

Let $\tau: B \rightarrow\left\langle\theta_{\ell r}\right\rangle$ be the homomorphism induced by the mapping $b \rightarrow \theta_{\ell r}$. Then $\tau$ is an extension function that produces an ideal extension of $\Gamma_{\ell r}^{*}$ by $B$ with a zero adjoined.

We denote by $\Gamma_{\ell r s}$ the ideal extension of $\Gamma_{\ell r}^{*}$ by $B^{0}$ determined by $\tau$, where $s$ is the order of $B$. 
It is time to summarize the main features of this and the preceding two sections. This provides a classification in terms of the finiteness of $\mathcal{L}$-classes or $\mathcal{R}$-classes. We say that a semigroup $S$ is of type $\Gamma, \Gamma_{\ell}, \Gamma_{\ell}^{\prime}$ (the dual of $\Gamma_{\ell}$ ), $\Gamma_{\ell r}$ or $\Gamma_{\ell r s}$, where $\ell, r, s$ are positive integers, if it is isomorphic to one of these semigroups for some choice of parameters.

THEOREM 5.2. Let $S$ be a completely regular semigroup generated by $\{a, b\}$, where $a<b^{0}$.

(i) $S$ is of type $\Gamma$ if and only if $S$ has an infinite number of $\mathcal{L}$-classes and $\mathcal{R}$-classes.

(ii) $S$ is of type $\Gamma_{\ell}$ if and only if $S$ has an infinite number of $\mathcal{L}$-classes and $\ell+1$ $\mathcal{R}$-classes.

(iii) $S$ is of type $\Gamma_{\ell}^{\prime}$ if and only if $S$ has $\ell+1 \mathcal{L}$-classes and an infinite number of $\mathcal{R}$-classes.

(iv) $S$ is of type $\Gamma_{\ell r s}$ if and only if $S$ has $r+1 \mathcal{L}$-classes, $\ell+1 \mathcal{R}$-classes, and $s$ is the order of the group of units of $S$.

\section{The case of $\Gamma_{\infty}$}

We now construct a special case of the semigroup $\Gamma$ in Section 3 as follows. Let $H=\left\{h_{n} \mid n \in \mathbb{Z}\right\}$, where $h_{m}=h_{n}$ only if $m=n$. Denote by $F H$ the free group on $H$. We follow the construction of $\Gamma$ with the following change of notation:

$$
\begin{gathered}
G \rightarrow F H, \quad \Gamma \rightarrow \Gamma_{\infty}, \quad \Gamma^{*} \rightarrow \Gamma_{\infty}^{*}, \\
\theta=(\alpha, \varphi ; \psi, \beta) \rightarrow \kappa=(\xi, \sigma ; \tau, \eta), \\
g_{n} \rightarrow h_{n}, \quad p_{n m} \rightarrow q_{n m},
\end{gathered}
$$

so that

$$
\Gamma_{\infty}^{*}=\mathcal{M}(\mathbb{Z}, F H, \mathbb{Z} ; Q), \quad \Gamma_{\infty}=\left(\Gamma_{\infty}^{*}, \kappa\right),
$$

and all formulae introduced in Section 3 are now assumed to be valid in the new notation.

Next let $S$ be a completely regular semigroup generated by a set $\{e, b\}$, where $e<b^{0}$. According to Theorem 5.2, $S$ is of one of the types $\Gamma, \Gamma_{\ell}, \Gamma_{\ell}^{\prime}, \Gamma_{\ell r s}$. We wish to construct a unique homomorphism of $\Gamma_{\infty}$ onto $S$. We may thus assume that $S$ is an ideal extension of $S_{0}=\mathcal{M}(I, G, \Lambda ; P)$ by $S_{1}^{0}$, where $S_{1}=\langle b\rangle$, the cyclic group generated by $b$ whose identity $b^{0}$ is the identity element of $S$. Further

$$
\begin{gathered}
I=\Lambda=\mathbb{Z} \quad \text { if } S \text { is of type } \Gamma, \\
I=I_{\ell}, \Lambda=\mathbb{Z} \quad \text { if } S \text { is of type } \Gamma_{\ell}, \\
I=\mathbb{Z}, \Lambda=\Lambda_{\ell} \quad \text { if } S \text { is of type } \Gamma_{\ell}^{\prime}, \\
I=I_{\ell}, \Lambda=\Lambda_{r} \quad \text { if } S \text { is of type } \Gamma_{\ell r s}, \text { where } s=o(b) .
\end{gathered}
$$

Note that the formulae for $p_{n m}$ for the case of $\Gamma$ remain valid for the cases of $\Gamma_{\ell}, \Gamma_{\ell}^{\prime}$, and $\Gamma_{\ell r s}$, and thus may be adopted here for $S$; see the converse part in Section 3. 
Since $F H$ is a free group, the mapping $h_{n} \rightarrow g_{n}$ extends uniquely to a homomorphism $\omega$ of $F H$ onto $G$. It follows that the mapping

$$
\chi_{0}:(m, h, n) \rightarrow(\bar{m}, h \omega, \bar{n}) \quad \forall(m, h, n) \in \Gamma_{\infty}^{*}
$$

is a homomorphism of $\Gamma_{\infty}^{*}$ onto $S_{0}$, where $0 \leq \bar{m}<\ell, \bar{m} \equiv m \bmod \ell$ in the case of $\Gamma_{\ell}$, and similarly for the other cases.

Next let $\chi_{1}:\langle\kappa\rangle \rightarrow S_{1}$ be the unique homomorphism that extends the mapping $\kappa \rightarrow b$. Now let $\chi=\chi_{0} \cup \chi_{1}$.

We have adopted above the same formulae for $q_{n m}, \sigma$ and $\tau$ as those defined in Section 3 for $p_{n m}, \varphi$ and $\psi$ respectively. In particular, Lemma 3.3 is applicable to $\sigma$ and $\tau$, that is, with the same formulae for $\sigma_{k}$ and $\tau_{k}$ as for $\varphi_{k}$ and $\psi_{k}$, with $h_{n}$ replacing $g_{n}$. Taking all this into account yields

$$
\begin{aligned}
\left(\kappa^{k}(m, g, n)\right) \chi & =\left(k+m,\left(\sigma_{k} m\right) g, n\right) \chi_{0} \\
& =\left(\overline{k+m},\left(\left(\varphi_{k} m\right) g\right) \omega, \bar{n}\right) \\
& =\left(\overline{k+m},\left(\sigma_{k} m\right) \omega(g \omega), \bar{n}\right) \\
& =\left(\overline{k+m},\left(\varphi_{k} \bar{m}\right)(g \omega), \bar{n}\right) \\
& =b^{k}(\bar{m}, g \omega, \bar{n})=\left(\theta^{k} \chi_{1}\right)(m, g, n) \chi_{0} \\
& =\left(\theta^{k} \chi\right)(m, g, n) \chi
\end{aligned}
$$

and similarly $\left((m, g, n) \theta^{k}\right) \chi=(m, g, n) \chi\left(\theta^{k} \chi\right)$. Therefore $\chi$ is a homomorphism. Since both $\chi_{0}$ and $\chi_{1}$ are surjective, so is $\chi$. Also $\chi$ extends the mapping $a \rightarrow e$, $\theta \rightarrow b$. Now $\{a, \theta\}$ generates $\Gamma_{\infty}$, so $\chi$ is the unique extension of this mapping.

We have thus proved the first part of the following result.

LEMMA 6.1. Let $S$ be a completely regular semigroup generated by $\{e, b\}$. If $e<$ $b^{0}$, then the mapping $a \rightarrow e, \theta \rightarrow b$ extends uniquely to a homomorphism of $\Gamma_{\infty}$ onto $S$. If $e=b^{0}$, then $S=\langle b\rangle$ and the mapping $a \rightarrow e, \theta \rightarrow b$ extends uniquely to a homomorphism of $\Gamma_{\infty}$ onto $S$.

PROOF. The mapping in the second part of the lemma is simply

$$
a \rightarrow e, \quad \theta^{n} \rightarrow b^{n} \quad \forall n \in \mathbb{Z},
$$

and we are done.

We denote the relatively free completely regular semigroup generated by a set $A$ subject to a set of relations $R$ by $\langle A \mid R\rangle$. We have thus established the next result.

THEOREM 6.2. $\Gamma_{\infty} \cong\left\langle e, b \mid e \leq b^{0}\right\rangle$.

Theorem 5.2 provides a classification of completely regular semigroups $S$ generated by $\{e, b\}$, where $e<b^{0}$. Theorem 6.2 characterizes a 'free-type' of such a semigroup. We now use these results to characterize such semigroups in two ways. 
THEOREM 6.3. The following conditions on a completely regular semigroup $S$ are equivalent.

(i) $S$ is generated by $\{e, b\}$, where $e<b^{0}$.

(ii) $S$ is isomorphic to some $\Gamma, \Gamma_{\ell}, \Gamma_{\ell}^{\prime}, \Gamma_{\ell r s}$.

(iii) $S$ is a homomorphic image of $\Gamma_{\infty}$ and has two completely simple components.

Note that Theorem 5.2 classifies these semigroups according to the finiteness of $\mathcal{L}$-classes and $\mathcal{R}$-classes, whereas Theorem 6.2 provides the semigroup $\left\langle e, b \mid e \leq b^{0}\right\rangle$, thereby supplementing Theorem 6.3 with additional information.

\section{The general case}

We are now interested in 2-generated completely regular monoids. So far we have classified completely regular semigroups $S$ generated by $\{e, b\}$, where $e<b^{0}$. This makes $b^{0}$ the identity of $S$, and $S$ has two components: a completely simple ideal $S_{0}$ and a cyclic group $S_{1}$.

The case where $S$ is generated by $\{a, b\}$ and $a^{0}<b^{0}$ may be obtained from the case in which $a$ is an idempotent studied so far quite simply, for it suffices to add one generator, $g$ say, to the generating set of $G$, in all cases. Instead of letting $a=(0,1,0)$, we set $a=(0, g, 0)$ and the entire discussion goes through. This takes care of the case when $\{a, b\}$ is a set of generators and $a^{0}<b^{0}$.

The case when $a^{0}=b^{0}$ amounts to a group generated by the set $\{a, b\}$.

This takes care of the case where $\{a, b\}$ is a set of generators and $a^{0} \leq b^{0}$.

Conversely, let $S$ be a completely regular monoid generated by the set $\{a, b\}$, and let $S=\left(Y ; S_{\alpha}\right)$. Since $S$ is a monoid, so is $Y$ and thus $|Y| \leq 2$. If $|Y|=1$, then $S$ must be a group, so that $a^{0}=b^{0}$. Hence $S$ is a 2-generator group. Suppose that $|Y|=2$; say $Y=\{0,1\}$. We may assume that $a \in S_{0}$ and $b \in S_{1}$. Then $b$ generates $S_{1}$, so that $S_{1}$ is a cyclic group. Its identity is $b^{0}$, which then must be the identity of $S$. This entails that $a^{0}<b^{0}$, the case discussed above.

The alteration described in the second paragraph above, namely the transition from $a<b^{0}$ to $a^{0}<b^{0}$, suggests the following notation. In the sequence of semigroups

$$
\Gamma, \Gamma_{\ell}, \Gamma_{\ell}^{\prime}, \Gamma_{\ell r s}, \Gamma_{\infty}
$$

we write $\Sigma$ for each $\Gamma$ to denote the case where we adjoin one generator to the generating set of $G$ in the construction of the semigroups in (7.1). We have thus arrived at the following classification of 2-generator completely regular monoids.

THEOREM 7.1. The following conditions on a completely regular semigroups are equivalent.

(i) $S$ is a 2-generator monoid.

(ii) $S$ is isomorphic to some $\Sigma, \Sigma_{\ell}, \Sigma_{\ell}^{\prime}, \Sigma_{\ell r s}$ or is a 2-generator group.

(iii) $S$ is a homomorphic image of $\Sigma_{\infty}$. 


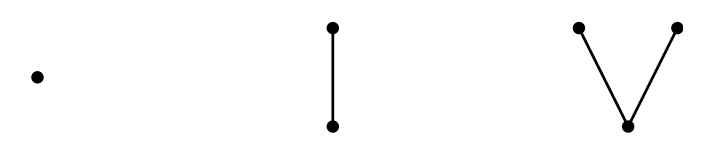

FIGURE 1. Three cases.

Corollary 7.2. Let $X=\{a, b\}$ and define a mapping $\sigma: X \rightarrow \Sigma_{\infty}$ by letting $b \sigma=b$ and $a \sigma=(0, g, 0)$. Then $\left(\sigma, \Sigma_{\infty}\right)$ is a free completely regular monoid on X. Moreover, $\Sigma_{\infty} \cong\left\langle a, b \mid a^{0} \leq b^{0}\right\rangle$.

One may wonder how far we are from classifying all 2-generator completely regular semigroups. For such semigroups, $S=\left(Y ; S_{\alpha}\right)$, where $Y$ has one of the diagrams in Figure 1.

In the first case, we are dealing with a completely simple semigroup with one of the cases: group, left group, right group, rectangular group, nonorthodox completely simple semigroup.

In the second case, the lower component would be a completely simple semigroup, and the upper a cyclic group. The ideal extension would be determined by a quadruple $(\alpha, \varphi ; \psi, \beta)$ as in the case already considered, but neither $\alpha$ nor $\beta$ need be permutations.

The third case would have two upper components cyclic groups, and the lower a completely simple semigroup. There would be an ideal extension of a completely simple semigroup by an orthogonal sum of two cyclic groups with a zero adjoined.

Recall that Clifford in [1] constructed a concrete copy of a free completely regular semigroup on two generators. This may help in classifying 2-generator completely regular semigroups. The above is merely a general program.

\section{References}

[1] A. H. Clifford, 'The free completely regular semigroup on a set', J. Algebra 59 (1979), 434-451.

[2] M. Petrich, Introduction to Semigroups (Merrill, Columbus, 1973).

[3] M. Petrich, 'A condition on the natural order for regular semigroups', Comm. Algebra 30 (2002), 517-542.

[4] M. Petrich, 'Characterizing cryptogroups with a finite number of $\mathcal{H}$-classes in each $\mathcal{D}$-class by their subsemigroups', Ann. Mat. Pura Appl. 187 (2008), 119-136.

[5] M. Petrich and N. R. Reilly, Completely Regular Semigroups (Wiley, New York, 1999). 